\title{
Budgetmitverantwortung versus Globalbudget
}

\section{Jörg Fritschi}

Präsident Dachverband Schweizer Ärztenetze medswiss.net

Korrespondenz: Dr. med. Jörg Fritschi Kleinbuholz 3 CH-6012 Obernau medswiss.net[at]hin.ch
Immer wieder wird die Budgetmitverantwortung gleichgesetzt mit einem Globalbudget.

Das ist ein Irrtum! Bei der Budgetmitverantwortung bleibt das Budget ein virtuelles Kostenziel. Der Betrag wird nicht ausbezahlt. Auch im Rahmen der Budgetmitverantwortung wird der weit überwiegende Anteil des ärztlichen Einkommens durch die Einzelleistungsvergütung erzielt. Das Schlüsselwort der Budgetmitverantwortung heisst Versorgungsausgleich. Das Globalbudget weist keines dieser Elemente auf.

\section{Versorgungsausgleich}

Unter- und Überversorgung sind gefährlich und nutzlos. Ein Globalbudget animiert zur Unterversorgung. Die alleinige Einzelleistungsverrechnung animiert zur Überversorgung und zur Mengenausweitung.

Die Budgetmitverantwortung gleicht beide Tendenzen aus: Sie kombiniert die Budgetpflicht eines Ärztenetzes mit der Einzelleistungsverrechnung seiner Mitglieder. Über- und Unterversorgung werden automatisch ausgeglichen (Abb. 1).

Ohne die Einzelleistungsverrechnung driftet Managed Care in die Richtung des Globalbudgets. Die Basis der Einzelleistungsverrechnung ist die Vertragspflicht der Versicherer bezüglich Zulassung und Tarif. Diese muss zwingend beibehalten werden. Alle diese Elemente sind im Schweizer Managed-CareModell enthalten: Swiss Managed Care!

Abbildung 2

Berechnung der Budgetmitverantwortung.

\section{Budgetmitverantwortung Berechnung}

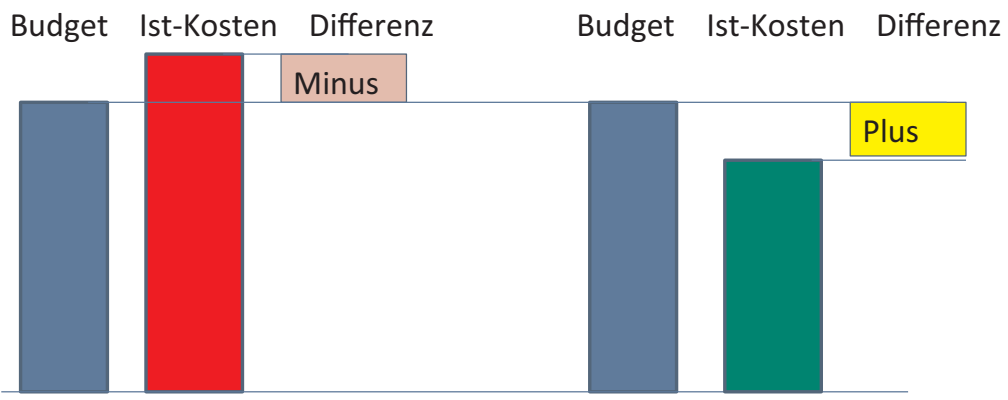

Minus und Plus werden zwischen Versicherer und Ärztenetz aufgeteilt $=$ Beteiligung $=$ Bonus/Malus, symmetrisch oder asymmetrisch verteilt

(Achtung: Grössenverhältnisse sind nur symbolisch)

Abbildung 1

Budgetmitverantwortung $=$ Versorgungsausgleich .

Budgetmitverantwortung Versorgungsausgleich

\begin{tabular}{|c|c|}
\hline $\begin{array}{c}\text { Einzel- } \\
\text { leistungs- } \\
\text { vergütung }\end{array}$ & $\begin{array}{c}\text { Alleiniges } \\
\text { Budget }\end{array}$ \\
\hline
\end{tabular}

Ausgleich Überversorgung und Unterversorgung

Versorgungsausgleich, damit Versorgungssicherheit Bedingung:

Einzelleistungsvergütung und Budget dürfen nicht von denselben Vertragspartnern ausgehandelt werden.

\section{Berechnung der Budgetmitverantwortung}

Im Rahmen eines Globalbudgets steht nur der budgetierte Betrag zur Verfügung.

Nicht so bei der Budgetmitverantwortung: Alle ärztlichen Leistungen werden mit der Einzelleistungsverrechnung abgerechnet. Der Budgetbetrag stellt lediglich ein virtuelles Kostenziel dar. Der budgetierte Betrag wird nicht ausbezahlt. Wird das Kostenziel über- oder unterschritten, wird die Differenz - und nur diese - von den beiden Partnern aufgeteilt. Versicherung und Ärztenetz tragen die Verantwortung gemeinsam: Budgetmitverantwortung (Abb. 2)!

\section{Managed Care ist Teamarbeit}

Die Gesetzesvorlage Managed Care sieht vor, dass die integrierenden Versorgungsnetze eine verhandelbare Budgetmitverantwortung übernehmen. Nur dann profitieren die Patientinnen und Patienten vom reduzierten Selbstbehalt. Wer Netzerfahrung hat, weiss, dass die Budgetmitverantwortung nichts Gefährliches ist. Ein ruinöses Budget führt zur Vertragsauflösung. An einer Vertragskündigung hat niemand Interesse, weder die Versicherer noch die Behörden.

Ein wichtiger Aspekt von Managed Care bleibt oft unbeachtet: Managed Care ist eine Plattform der Kommunikation. Die Spannungsfelder «medizinischer Fortschritt», «Altersentwicklung» und «Anspruchshaltung» auf der einen und «begrenzte Ressourcen» auf der anderen Seite lassen sich durch Teamarbeit besser lösen.

Damit ist Managed Care keine Bedrohung für die Schweizer Ärzteschaft, sondern eine Chance! 